PROCEEDINGS OF THE

AMERICAN MATHEMATICAL SOCIETY

Volume 135, Number 8, August 2007, Pages 2505-2517

S 0002-9939(07)08729-1

Article electronically published on March 21, 2007

\title{
FIXED POINT THEOREMS IN ORDERED ABSTRACT SPACES
}

\author{
JUAN J. NIETO, RODRIGO L. POUSO, AND ROSANA RODRÍGUEZ-LÓPEZ
}

(Communicated by Joseph A. Ball)

\begin{abstract}
We extend some fixed point theorems in $L$-spaces, obtaining extensions of the Banach fixed point theorem to partially ordered sets.
\end{abstract}

\section{Preliminaries}

In 1], some results on the existence of fixed points in partially ordered sets are presented and applied to the resolution of matrix equations. The main result in [1] is:

Theorem 1.1 ([1]). Let $X$ be a partially ordered set such that every pair $x, y \in X$ has an upper and a lower bound. Let $d$ be a metric on $X$ such that $(X, d)$ is complete. Let $f: X \rightarrow X$ be a continuous and monotone (either order-preserving or order-reversing) operator. Suppose that the following assertions hold:

1. there exists $k \in(0,1)$ with $d(f(x), f(y)) \leq k d(x, y), \forall x \geq y$,

2. there exists $x_{0} \in X$ with $x_{0} \leq f\left(x_{0}\right)$ or $x_{0} \geq f\left(x_{0}\right)$.

Then $f$ has a unique fixed point $x^{*} \in X$, and for each $x \in X,\left(f^{n}(x)\right)_{n \in \mathbb{N}} \longrightarrow x^{*}$.

In [2, 3] the existence of a unique fixed point for mappings in partially ordered sets is studied, giving some applications to the existence of a unique solution for a first-order ordinary differential equation with periodic boundary conditions. The authors improved Theorem 1.1 in the following directions:

Theorem $1.2([2])$. Let $(X, \leq)$ be a partially ordered set and suppose that there exists a metric $d$ in $X$ such that $(X, d)$ is a complete metric space.

Let $f: X \rightarrow X$ be a monotone nondecreasing mapping such that there exists $k \in[0,1)$ with

$$
d(f(x), f(y)) \leq k d(x, y), \forall x \geq y .
$$

Suppose that either $f$ is continuous or $X$ is such that

$$
\text { if a nondecreasing sequence }\left(x_{n}\right)_{n \in \mathbb{N}} \rightarrow x \text { in } X \text {, then } x_{n} \leq x, \forall n \text {. }
$$

If there exists $x_{0} \in X$ with $x_{0} \leq f\left(x_{0}\right)$, then $f$ has a fixed point.

Received by the editors October 20, 2005 and, in revised form, March 20, 2006

2000 Mathematics Subject Classification. Primary 47H10.

Key words and phrases. Fixed point, partially ordered set, $L$-spaces.

This research was partially supported by Ministerio de Educación y Ciencia and FEDER, project MTM2004-06652-C03-01, and by Xunta de Galicia and FEDER, project PGIDIT05PXIC20702PN. 
Theorem $1.3(2])$. Let $(X, \leq)$ be a partially ordered set and suppose that there exists a metric $d$ in $X$ such that $(X, d)$ is a complete metric space.

Let $f: X \rightarrow X$ be a monotone nondecreasing mapping such that there exists $k \in[0,1)$ with $d(f(x), f(y)) \leq k d(x, y), \forall x \geq y$. Suppose that either $f$ is continuous or $X$ is such that

$$
\text { if a nonincreasing sequence }\left(x_{n}\right)_{n \in \mathbb{N}} \rightarrow x \text { in } X \text {, then } x \leq x_{n}, \forall n \text {. }
$$

If there exists $x_{0} \in X$ with $x_{0} \geq f\left(x_{0}\right)$, then $f$ has a fixed point.

Uniqueness of a fixed point and global convergence of the method of successive approximations can be obtained adding the hypothesis

$$
\text { every pair of elements of } X \text { has a lower bound or an upper bound. }
$$

This condition is equivalent to

for every $x, y \in X$, there exists $z \in X$ which is comparable to $x$ and $y$.

Theorem 1.4 (Theorem 4 in [3]). Let $(X, \leq)$ be a partially ordered set satisfying (1.3) and suppose that there exists a metric $d$ in $X$ such that $(X, d)$ is a complete metric space.

Let $f: X \rightarrow X$ be a nonincreasing function such that there exists $k \in[0,1)$ with $d(f(x), f(y)) \leq k d(x, y), \forall x \geq y$. Suppose also that either $f$ is continuous or $X$ is such that

$$
\begin{aligned}
& \text { if }\left(x_{n}\right)_{n \in \mathbb{N}} \rightarrow x \text { is a sequence in } X \text { whose consecutive terms are } \\
& \text { comparable, then there exists a subsequence }\left(x_{n_{k}}\right)_{k \in \mathbb{N}} \\
& \text { of }\left(x_{n}\right)_{n \in \mathbb{N}} \text { such that every term is comparable to the limit } x .
\end{aligned}
$$

If there exists $x_{0} \in X$ with $x_{0} \leq f\left(x_{0}\right)$ or $x_{0} \geq f\left(x_{0}\right)$, then $f$ has a unique fixed point.

Theorem 1.5 (Theorem 7 in [3]). Let $(X, \leq)$ be a partially ordered set and suppose that (1.3) holds and that there exists a metric $d$ in $X$ such that $(X, d)$ is a complete metric space. Let $f: X \rightarrow X$ be such that $f$ maps comparable elements into comparable elements, that is,

$$
x, y \in X, x \leq y \Longrightarrow\left\{\begin{array}{c}
f(x) \leq f(y) \\
\text { or } \\
f(x) \geq f(y)
\end{array}\right.
$$

and such that there exists $k \in[0,1)$ with $d(f(x), f(y)) \leq k d(x, y), \forall x \geq y$. Suppose that either $f$ is continuous or $X$ is such that condition (1.4) holds.

If there exists $x_{0} \in X$ with $x_{0}$ comparable to $f\left(x_{0}\right)$, then $f$ has a unique fixed point $\bar{x}$. Moreover, $\forall x \in X, \lim _{n \rightarrow+\infty} f^{n}(x)=\bar{x}$.

On the other hand, in [4, the results of [1] are also extended, proving some general fixed point theorems in ordered $L$-spaces. Then, the authors apply the results to the resolution of matrix equations and differential systems.

In this paper, we improve the above-mentioned results to the case of $L$-spaces, extending the results in [4] by relaxing some hypotheses and allowing some more general types of continuity for the mapping. 


\section{BASIC CONCEPTS ON $L$-SPACES}

In our results, we use the formulation of 4 . Recall the following concepts.

Let $X$ be a nonempty set and let $f: X \longrightarrow X$ be an operator. We denote the successive iterations of $f$ as $f^{0}=1_{X}, f^{1}=f, f^{n}=f \circ f^{n-1}$, for $n \in \mathbb{N}, n \geq 2$.

Definition 2.1. The set of all nonempty invariant subsets of $f$ is

$$
I(f):=\{Y \subset X: f(Y) \subseteq Y\},
$$

and the set of fixed points of $f$ is $F_{f}:=\{x \in X: x=f(x)\}$.

We denote by $s(X)$ the set of sequences in $X$, that is,

$$
s(X):=\left\{\left(x_{n}\right)_{n \in \mathbb{N}}: x_{n} \in X, n \in \mathbb{N}\right\} .
$$

Fréchet introduced in [5] the notion of $L$-space as follows:

Definition 2.2. An $L$-space is a triple $(X, c(X), \operatorname{Lim})$, where $X$ is a set, $c(X) \subseteq$ $s(X)$ is a family of sequences of elements of $X$, and $\operatorname{Lim}: c(X) \rightarrow X$ is a mapping having the following two properties:

i): If $x_{n}=x$, for all $n \in \mathbb{N}$, then $\left(x_{n}\right)_{n \in \mathbb{N}} \in c(X)$ and $\operatorname{Lim}\left(x_{n}\right)_{n \in \mathbb{N}}=x$.

ii): If $\left(x_{n}\right)_{n \in \mathbb{N}} \in c(X)$ and $\operatorname{Lim}\left(x_{n}\right)_{n \in \mathbb{N}}=x$, then for all subsequences $\left(x_{n_{i}}\right)_{i \in \mathbb{N}}$ of $\left(x_{n}\right)_{n \in \mathbb{N}}$ we have that $\left(x_{n_{i}}\right)_{i \in \mathbb{N}} \in c(X)$ and $\operatorname{Lim}\left(x_{n_{i}}\right)_{i \in \mathbb{N}}=x$.

The elements of $c(X)$ are called convergent sequences and $x:=\operatorname{Lim}\left(x_{n}\right)_{n \in \mathbb{N}}$ is the limit of the sequence, also written $x_{n} \rightarrow x$ as $n \rightarrow+\infty$. An $L$-space is denoted by $(X, \rightarrow)$.

The attractor basin of $f$ with respect to an element $x^{*} \in X$ is

$$
A_{f}\left(x^{*}\right):=\left\{x \in X: f^{n}(x) \rightarrow x^{*}, \text { as } n \rightarrow+\infty\right\} .
$$

Definition 2.3. $f: X \longrightarrow X$ is said to be orbitally continuous if $x \in X$, and $f^{n(i)}(x) \rightarrow a$, as $i \rightarrow+\infty$, imply that $f^{n(i)+1}(x) \rightarrow f(a)$, as $i \rightarrow+\infty$.

Definition 2.4. Let $(X, \leq)$ be a partially ordered set and

$$
X_{\leq}:=\{(x, y) \in X \times X: x \leq y \text { or } y \leq x\} .
$$

For $x, y \in X$, with $x \leq y$, we denote it by

$$
[x, y]_{\leq}:=\{z \in X: x \leq z \leq y\} .
$$

If $f: X \longrightarrow X$,

$$
(L F)_{f}:=\{x \in X: x \leq f(x)\},(U F)_{f}:=\{x \in X: x \geq f(x)\}
$$

are, respectively, the lower and the upper fixed point sets of $f$.

For $f: X \longrightarrow X, g: Y \longrightarrow Y$, the cartesian product of $f$ and $g$ is defined as

$$
f \times g: X \times Y \longrightarrow X \times Y,(f \times g)(x, y)=(f(x), g(y)) \text {. }
$$

Definition 2.5. For $X$ a nonempty set, $(X, \rightarrow, \leq)$ is an ordered $L$-space if

i): $(X, \rightarrow)$ is an $L$-space.

ii): $(X, \leq)$ is a partially ordered set.

iii): If $\left(x_{n}\right)_{n \in \mathbb{N}} \rightarrow x,\left(y_{n}\right)_{n \in \mathbb{N}} \rightarrow y$ and $x_{n} \leq y_{n}$, for each $n \in \mathbb{N}$, then $x \leq y$.

The results we prove are about the existence of a fixed point for an operator $f$ such that the sequences of successive iterations starting at an arbitrary element of the space converge to that fixed point (the unique fixed point). In other words, we try to determine if a certain operator is a Picard operator. 
Definition 2.6. Let $(X, \rightarrow)$ be an $L$-space. An operator $f: X \rightarrow X$ is, by definition, a Picard operator (briefly PO) if:

i): $F_{f}$ is a singleton-set, say $F_{f}=\left\{x^{*}\right\}$,

ii): $\left(f^{n}(x)\right)_{n \in \mathbb{N}} \rightarrow x^{*}$, as $n \rightarrow+\infty$, for all $x \in X$.

For more details, see [4] and the references therein.

\section{Fixed point theorems in $L$-SPACES}

According to the previous notation, Theorem [1.5 (Theorem 7 in [3]) can be written in the following terms:

Theorem 3.1. Let $(X, d)$ be a metric space equipped with a partial ordering $\leq$ and let $f: X \longrightarrow X$ be an operator. Suppose that:

a): Condition (1.3) holds, that is, for every $x, y \in X$, there exists $z \in X$ such that $(x, z),(y, z) \in X_{\leq}$.

b): $f$ maps comparable elements into comparable elements, that is,

$$
X_{\leq} \in I(f \times f)
$$

c): One of the following conditions is satisfied:

c.1): $f:(X, d) \longrightarrow(X, d)$ is orbitally continuous or

c.2): condition (1.4) holds, that is,

if $\left(x_{m}\right)_{m \in \mathbb{N}} \rightarrow x$ is a sequence in $X$ such that $\left(x_{m}, x_{m+1}\right) \in X_{\leq}, \forall m \in \mathbb{N}$,

then there exists a subsequence $\left(x_{m_{k}}\right)_{k \in \mathbb{N}}$ of $\left(x_{m}\right)_{m \in \mathbb{N}}$

such that $\left(x_{m_{k}}, x\right) \in X_{\leq}, \forall k \in \mathbb{N}$.

d): The metric $d$ is complete.

e): There exists $x_{0} \in X$ with $\left(x_{0}, f\left(x_{0}\right)\right) \in X_{\leq}$.

f): There exists $k \in[0,1)$ with $d(f(x), f(y)) \leq k d(x, y), \forall(x, y) \in X_{\leq}$.

Then $f: X \longrightarrow X$ is a $P O$.

Proof. For completeness and to use the new notation, we sketch the proof. Suppose that $f\left(x_{0}\right) \neq x_{0}$. Using e) and $\left.\mathrm{b}\right)$, we prove, by induction, that $\left(f^{n}\left(x_{0}\right), f^{n+1}\left(x_{0}\right)\right) \in$ $X_{\leq}$, for every $n \in \mathbb{N}$. Condition $\mathrm{f}$ ) implies that $\left(f^{n}\left(x_{0}\right)\right)_{n \in \mathbb{N}}$ is a Cauchy sequence in $X$. By d), there exists $x^{*} \in X$ such that $\left(f^{n}\left(x_{0}\right)\right)_{n \in \mathbb{N}} \longrightarrow x^{*}$, as $n \rightarrow+\infty$. Let $x \in X$ be arbitrary, and distinguish two cases:

- If $\left(x, x_{0}\right) \in X_{\leq}$, then $\left(f^{n}(x), f^{n}\left(x_{0}\right)\right) \in X_{\leq}$, for every $n \in \mathbb{N}$ and

$$
\begin{aligned}
d\left(f^{n}(x), x^{*}\right) \leq d\left(f^{n}(x), f^{n}\left(x_{0}\right)\right)+ & d\left(f^{n}\left(x_{0}\right), x^{*}\right) \\
& \leq k^{n} d\left(x, x_{0}\right)+d\left(f^{n}\left(x_{0}\right), x^{*}\right) \rightarrow 0, n \rightarrow+\infty .
\end{aligned}
$$

- If $\left(x, x_{0}\right) \notin X_{\leq}$, by a), there exists $z \in X$ such that $(x, z),\left(x_{0}, z\right) \in X_{\leq}$. Similarly to the previous item, we obtain $f^{n}(z) \rightarrow x^{*}$ and $f^{n}(x) \rightarrow x^{*}$.

If $f$ is orbitally continuous, then $x^{*}$ is obviously a fixed point of $f$. Under the hypothesis c.2), take into account that $\left(f^{n}\left(x_{0}\right)\right)_{n \in \mathbb{N}}$ is a sequence such that

$$
\left(f^{n}\left(x_{0}\right)\right) \rightarrow x^{*}, \quad\left(f^{n}\left(x_{0}\right), f^{n+1}\left(x_{0}\right)\right) \in X_{\leq}, \forall n \in \mathbb{N},
$$

then there exists $\left(f^{n_{k}}\left(x_{0}\right)\right)_{k \in \mathbb{N}}$ a subsequence of $\left(f^{n}\left(x_{0}\right)\right)_{n \in \mathbb{N}}$ such that

$$
\left(f^{n_{k}}\left(x_{0}\right), x^{*}\right) \in X_{\leq}, \forall k \in \mathbb{N},
$$


then

$$
\begin{gathered}
d\left(x^{*}, f\left(x^{*}\right)\right) \leq d\left(x^{*}, f^{n_{k}+1}\left(x_{0}\right)\right)+d\left(f\left(f^{n_{k}}\left(x_{0}\right)\right), f\left(x^{*}\right)\right) \\
\leq d\left(x^{*}, f^{n_{k}+1}\left(x_{0}\right)\right)+k d\left(f^{n_{k}}\left(x_{0}\right), x^{*}\right) \stackrel{k \rightarrow+\infty}{\longrightarrow} 0 .
\end{gathered}
$$

If $f\left(x_{0}\right)=x_{0}, x_{0}$ plays the role of $x^{*}$.

Note that condition c.2) is trivially fulfilled for totally ordered spaces, where Theorem 3.1 is the classical Banach contraction principle.

Obviously, if $f$ is monotone, then condition b) holds. This result improves Theorem 1.1 (Theorem 2.1 in [1) and Theorem 4.3 in 4. Indeed, we present an alternative condition to orbital continuity of $f$ and relax another hypothesis from 4, where the authors considered the more restrictive condition

$\tilde{a})$ : For every $x, y \in X$, there exist $m(x, y), M(x, y) \in X$ such that $(x, y) \in$ $[m(x, y), M(x, y)]_{\leq}$.

For $L$-spaces equipped with a partial ordering $\leq$, we obtain the following result, which requires us to define previously the concept of orbitally $\leq$-continuous functions.

Definition 3.2. Let $(X, \rightarrow)$ be an $L$-space equipped with a partial ordering $\leq$. We say that $f:(X, \rightarrow) \longrightarrow(X, \rightarrow)$ is orbitally $\leq$-continuous if $x \in X$ and

$$
\begin{gathered}
f^{n(i)}(x) \rightarrow a, \\
\left(f^{n(i)}(x), a\right) \in X_{\leq}, \quad \forall i \in \mathbb{N},
\end{gathered}
$$

imply $f^{n(i)+1}(x) \rightarrow f(a)$.

Theorem 3.3. Let $(X, \rightarrow)$ be an $L$-space with a partial order $\leq$ and let $f: X \longrightarrow X$ be an operator. Suppose that:

A): For every $x, y \in X$, there exists $z \in X$ such that $(x, z),(y, z) \in X_{\leq}$.

B): There exist $x_{0}, x^{*} \in X$ such that $\left(f^{n}\left(x_{0}\right)\right)_{n \in \mathbb{N}} \rightarrow x^{*}$, as $n \rightarrow+\infty$.

C): One of the following conditions is satisfied:

C.1): $f:(X, \rightarrow) \longrightarrow(X, \rightarrow)$ is orbitally continuous or

C.2): $f:(X, \rightarrow) \longrightarrow(X, \rightarrow)$ is orbitally $\leq$-continuous and there exists $\left(f^{n_{k}}\left(x_{0}\right)\right)_{k \in \mathbb{N}}$, a subsequence of $\left(f^{n}\left(x_{0}\right)\right)_{n \in \mathbb{N}}$, such that $\left(f^{n_{k}}\left(x_{0}\right), x^{*}\right) \in$ $X_{\leq}, \forall k \in \mathbb{N}$.

D): If $(x, y) \in X_{\leq}$and $\left(f^{n}(x)\right)_{n \in \mathbb{N}} \rightarrow x^{*}$, as $n \rightarrow+\infty$, then $\left(f^{n}(y)\right)_{n \in \mathbb{N}} \rightarrow$ $x^{*}$, as $n \rightarrow+\infty$.

Then $f: X \longrightarrow X$ is a PO.

Proof. Suppose that $f\left(x_{0}\right) \neq x_{0}$. Using B), we get $\left(f^{n}\left(x_{0}\right)\right)_{n \in \mathbb{N}} \rightarrow x^{*}$.

From C.1), $x^{*}$ is a fixed point of $f$. In case C.2), there exists $\left(f^{n_{k}}\left(x_{0}\right)\right)_{k \in \mathbb{N}} \rightarrow$ $x^{*},\left(f^{n_{k}}\left(x_{0}\right), x^{*}\right) \in X_{\leq}, \forall k \in \mathbb{N}$. Then $\left(f^{n_{k}+1}\left(x_{0}\right)\right)_{k \in \mathbb{N}} \rightarrow f\left(x^{*}\right)$, but this is a subsequence of $\left(f^{n}\left(x_{0}\right)\right)_{n \in \mathbb{N}}$ and, therefore, $f\left(x^{*}\right)=x^{*}$.

Let $x \in X$ be arbitrary, and distinguish two cases:

- If $\left(x, x_{0}\right) \in X_{\leq}$, then, by B) and D), $\left(f^{n}(x)\right)_{n \in \mathbb{N}} \rightarrow x^{*}$.

- If $\left(x, x_{0}\right) \notin X_{\leq}$, by A), there exists $z \in X$ such that $(x, z),\left(x_{0}, z\right) \in X_{\leq}$; in consequence, by $\mathrm{D}), f^{n}(z) \rightarrow x^{*}$ and $f^{n}(x) \rightarrow x^{*}$.

If $f\left(x_{0}\right)=x_{0}$, then $x_{0}$ plays the role of $x^{*}$. 
Theorem 3.1 is a particular case of Theorem 3.3, since a) coincides with A); c.1) coincides with (.1); b), d), e), f) imply B); b), f) imply D); and b), c.2), e) imply the second part in C.2). Moreover, condition f) implies the orbital $\leq$-continuity of $f$. Indeed, if $x \in X$ and

$$
\begin{gathered}
f^{n(i)}(x) \rightarrow a \in X \\
\left(f^{n(i)}(x), a\right) \in X_{\leq}, \forall i \in \mathbb{N}
\end{gathered}
$$

then

$$
d\left(f^{n(i)+1}(x), f(a)\right) \leq k d\left(f^{n(i)}(x), a\right) \rightarrow 0,
$$

and $f^{n(i)+1}(x) \rightarrow f(a)$. Besides, our Theorem 3.3 extends Theorem 4.2 in 4 ] since the hypothesis about continuity of $f$ has been relaxed and we allow $f$ to be discontinuous.

For a more general formulation of fixed point results in $L$-spaces, we give the following definition:

Definition 3.4. Let $(X, \rightarrow)$ be an $L$-space and $U \subseteq X \times X$. We say that $f$ is orbitally $U$-continuous if $x \in X$ and

$$
\begin{gathered}
f^{n(i)}(x) \rightarrow a, \\
\left(f^{n(i)}(x), a\right) \in U, \quad \forall i \in \mathbb{N},
\end{gathered}
$$

imply $f^{n(i)+1}(x) \rightarrow f(a)$.

Theorem 3.5. Let $(X, \rightarrow)$ be an $L$-space and $U$ a symmetric subset of $X \times X$ such that $\Delta(X)$ (the diagonal of $X \times X$ ) is included in $U$. Let $f: X \longrightarrow X$ be an operator. Suppose that:

I): For every $x, y \in X$, there exists $z \in X$ such that $(x, z),(y, z) \in U$.

II): There exist $x_{0}, x^{*} \in X$ such that $x_{0} \in A_{f}\left(x^{*}\right)$.

III): $(x, y) \in U$ and $x \in A_{f}\left(x^{*}\right)$ imply that $y \in A_{f}\left(x^{*}\right)$.

Then $A_{f}\left(x^{*}\right)=X$. Moreover, if one of the following conditions hold:

IV.a): $f$ is orbitally continuous,

IV.b): $f$ is orbitally $U$-continuous and there exists $\left(f^{n_{k}}\left(x_{0}\right)\right)_{k \in \mathbb{N}}$, a subsequence of $\left(f^{n}\left(x_{0}\right)\right)_{n \in \mathbb{N}}$, such that $\left(f^{n_{k}}\left(x_{0}\right), x^{*}\right) \in U, \forall k \in \mathbb{N}$, then $F_{f}=\left\{x^{*}\right\}$.

Proof. Let $x \in X$ be arbitrary.

- If $\left(x, x_{0}\right) \in U$, then $x \in A_{f}\left(x^{*}\right)$, using II) and III).

- If $\left(x, x_{0}\right) \notin U$, by I), there exists $z \in X$ such that $(x, z),\left(x_{0}, z\right) \in U$. By II) and III), we get $x \in A_{f}\left(x^{*}\right)$.

It is evident that an element different from $x^{*}$ is not a fixed point of $f$. Under conditions IV.a) or IV.b), we prove that $x^{*}$ is a fixed point of $f$ (the unique fixed point of $f)$. If $f$ is orbitally continuous, then $f^{n+1}\left(x_{0}\right)=f\left(f^{n}\left(x_{0}\right)\right) \rightarrow f\left(x^{*}\right)$ and $f\left(x^{*}\right)=x^{*}$. Suppose that condition IV.b) holds. Then $\left(f^{n_{k}}\left(x_{0}\right)\right)_{k \in \mathbb{N}} \rightarrow x^{*}$, $\left(f^{n_{k}}\left(x_{0}\right), x^{*}\right) \in U, \forall k \in \mathbb{N}$. In consequence, by the orbital $U$-continuity of $f$, we obtain $\left(f^{n_{k}+1}\left(x_{0}\right)\right)_{k \in \mathbb{N}} \rightarrow f\left(x^{*}\right)$, obtaining, from the property ii) of $L$-spaces, $f\left(x^{*}\right)=x^{*}$.

Theorem 3.3 is a particular case of Theorem 3.5 taking $U=X_{\leq}$which is a symmetric subset of $X \times X$ containing the diagonal $\Delta(X)$. Note that orbital $\leq-$ continuity is precisely orbital $X_{\leq}$-continuity. Theorem 3.5 improves Lemma 4.1 in [4], since orbital continuity is not essential in our result. 
Next, we present some applications of the previous fixed point theorems.

Theorem 3.6. Let $(X, d)$ be a generalized metric space (in the sense that $d(x, y) \in$ $\mathbb{R}_{+}^{n}$ ) with a partial ordering $\leq$ and let $f: X \longrightarrow X$ be an operator. Suppose that hypotheses a)-e) in Theorem 3.1 hold and that

$\tilde{f})$ : There exists a matrix $A \in M_{n n}\left(\mathbb{R}_{+}\right)$with $A^{m} \rightarrow 0$ as $m \rightarrow+\infty$, such that

$$
d(f(x), f(y)) \leq A \cdot d(x, y), \forall(x, y) \in X_{\leq} .
$$

Then $f:(X, d) \longrightarrow(X, d)$ is a $P O$.

Theorem 3.7. Let $(X, F$, min, $\leq)$ be a probabilistic complete metric space with a partial ordering $\leq$, and let $f: X \longrightarrow X$ be an operator. Suppose that a), b), e) in Theorem 3.1 hold and

$\tilde{c})$ : One of the following conditions is satisfied:

- $f:(X, F, \min ) \longrightarrow(X, F$, min $)$ is orbitally continuous, or

- condition c.2) holds.

$\tilde{f}):$ There exists $k \in[0,1)$ with $F_{f(x), f(y)}(a \lambda) \geq F_{x, y}(\lambda), \forall(x, y) \in X_{\leq}, \lambda>0$.

Then $f: X \longrightarrow X$ is a PO.

These results extend Theorems 4.7 and 4.8 in [4. Analogous considerations can be made for complete Menger spaces with a partial ordering $\leq$, relaxing the corresponding hypotheses and improving Theorem 4.10 in [4].

For systems of nonlinear equations in $\mathbb{R}^{m}$, we can obtain a result more general than Theorem 5.3 in [4, since orbital continuity can be eliminated if $m=1$, obtaining the following result.

Theorem 3.8. Take the ordered L-space $\left(\mathbb{R}^{m}, \rightarrow, \leq\right)$ and let $f: \mathbb{R}^{m} \longrightarrow \mathbb{R}^{m}$ be an operator. Suppose that:

- $\mathbb{R}_{\leq}^{m} \in I(f \times f)$.

- One of the following conditions is satisfied: either $f: \mathbb{R}^{m} \longrightarrow \mathbb{R}^{m}$ is orbitally continuous, or condition c.2) holds for $X=\mathbb{R}^{m}$.

- There exists $x_{0} \in \mathbb{R}^{m}$ with $\left(x_{0}, f\left(x_{0}\right)\right) \in \mathbb{R}_{\leq}^{m}$.

- There exists a matrix $A \in M_{m m}\left(\mathbb{R}_{+}\right)$with $A^{n} \rightarrow 0$ as $n \rightarrow+\infty$, such that

$$
\left(\begin{array}{c}
\left\|f_{1}(x)-f_{1}(y)\right\| \\
\vdots \\
\left\|f_{m}(x)-f_{m}(y)\right\|
\end{array}\right) \leq A \cdot\left(\begin{array}{c}
\left\|x_{1}-y_{1}\right\| \\
\vdots \\
\left\|x_{m}-y_{m}\right\|
\end{array}\right), \forall x, y \in \mathbb{R}^{m}, x \leq y .
$$

Then the equation $x=f(x)$ has a unique solution $x^{*} \in \mathbb{R}^{m}$ and $f^{n}(x) \rightarrow x^{*}$ as $n \rightarrow+\infty$, for all $x \in \mathbb{R}^{m}$. by:

In the case of nondecreasing operators $f$ (see [2]), hypothesis c.2) can be replaced

$$
\text { if }\left(x_{n}\right)_{n \in \mathbb{N}} \rightarrow x \text { is monotone, then every } x_{n} \text { is comparable to the limit } x,
$$

which is valid, for instance, for $X=\mathbb{R}^{m}, X=C(I, \mathbb{R})$ the space of continuous functions $x: I \longrightarrow \mathbb{R}$, and $X=E^{1}$ the space of real fuzzy numbers [6]. In this case, orbital continuity can be eliminated. 


\section{Fixed POINT THEOREMS IN TOPOLOGICAL SPACES}

Plainly, every Hausdorff topological space is an $L$-space: if $X$ is a topological space it suffices to define $c(X)$ as the family of all convergent sequences and $\mathrm{Lim}$ as the mapping that sends every convergent sequence into its limit. Note that it is not only that Hausdorff topological spaces are $L$-spaces, but they can be naturally equipped with an $L$-space structure such that convergent sequences and their respective limits are the same from the topological and from the $L$-space points of view.

Not as obvious, every $L$-space is a topological space. More precisely: for an $L$-space $(X, c(X), \operatorname{Lim})$ there exists a topology in $X$, say $\tau_{L}$, such that every sequence $\left(x_{n}\right)_{n} \in c(X)$ converges to $\operatorname{Lim}\left(\left(x_{n}\right)_{n}\right)$ in the $\tau_{L}$ topology. To define such a topology it suffices to define a basis of open neighborhoods of each point as follows: for every $x \in X$ we define a basic open neighborhood of $x$ as any set of the form

$$
\bigcup_{\left(y_{n}\right)_{n} \in L i m^{-1}(\{x\})} \bigcup_{n=k\left(\left(y_{n}\right)_{n}\right)}^{\infty}\left\{y_{n}\right\} \quad \text { where } k: c(X) \rightarrow \mathbb{N},
$$

i.e., basic open neighborhoods of $x$ are sets which contain all terms of all sequences that converge to $x$ except, at most, a finite number of terms of each sequence. Finally we define the elements of $\tau_{L}$ as subsets of $X$ that contain a basic open neighborhood of each one of their points.

Obviously, if $\left(x_{n}\right)_{n} \in c(X)$ and $\operatorname{Lim}\left(\left(x_{n}\right)_{n}\right)=x$, then any open neighborhood of $x$, by its definition, contains all terms of $\left(x_{n}\right)_{n}$ except, at most, a finite number of them, i.e., $\left(x_{n}\right)_{n}$ tends to $x$ in the $\tau_{L}$ topology. However there might be more $\tau_{L}$-convergent sequences than $L$-convergent sequences, and this is impossible to avoid in general. This is due to the fact that certain sequences that belong to $c(X)$ may not belong to $c(X)$ anymore if we add a finite number of terms; moreover, it is possible that we could combine the terms of two elements of $c(X)$ that have the same limit to construct a new sequence that does not belong to $c(X)$. Fréchet pointed out that this is an inconvenience of $L$-spaces; see 5 . Furthermore $\tau_{L}$ is not necessarily a Hausdorff topology, so it might be possible to find one sequence with different limits.

As a conclusion we can say that an $L$-space can be equivalently defined as a triple $(X, c(X), \operatorname{Lim})$, where $X$ is a topological space, $c(X)$ is a certain family of convergent sequences, and Lim $: c(X) \rightarrow X$ is an operator that assigns one of its limits to each sequence in $c(X)$ and satisfies i) and ii) in Definition 2.2.

The next results are applicable, in particular, to not necessarily complete metric spaces.

Theorem 4.1. Let $X$ be a Hausdorff topological space with a partial order $\leq$ and let $f: X \longrightarrow X$ be an operator. Suppose that:

- $f$ is order-preserving.

- $f$ is orbitally monotone-continuous, that is, if $x \in X$ and $f^{n(i)}(x) \rightarrow$ a with $\left(f^{n(i)}(x)\right)_{i \in \mathbb{N}}$ monotone, then $f^{n(i)+1}(x) \rightarrow f(a)$.

- There exists $x_{0} \in X$ with $\left(x_{0}, f\left(x_{0}\right)\right) \in X_{\leq}$.

- $f$ maps monotone sequences into convergent sequences.

Then there exists at least one fixed point of $f$ in X. If A), D) in Theorem 3.3 hold, then $f$ is a PO. 
Proof. Since $\left(x_{0}, f\left(x_{0}\right)\right) \in X_{\leq}$and $f$ is nondecreasing, then $\left(f^{n-1}\left(x_{0}\right)\right)_{n \in \mathbb{N}}$ is a monotone sequence, so that $\left(f^{n}\left(x_{0}\right)\right)_{n \in \mathbb{N}}$ is a convergent sequence. Now,

$$
\left(f^{n}\left(x_{0}\right)\right)_{n \in \mathbb{N}} \rightarrow x^{*}
$$

is monotone, hence, by the orbital monotone-continuity of $f,\left(f^{n+1}\left(x_{0}\right)\right)_{n \in \mathbb{N}} \rightarrow$ $f\left(x^{*}\right)$. In consequence, $f\left(x^{*}\right)=x^{*}$.

On the other hand, let $x \in X$ be arbitrary.

- If $\left(x, x_{0}\right) \in X_{\leq}$, then $\left(f^{n}(x)\right)_{n \in \mathbb{N}} \rightarrow x^{*}$.

- If $\left(x, x_{0}\right) \notin X_{\leq}$, by a), there exists $z \in X$ such that $(x, z),\left(x_{0}, z\right) \in X_{\leq}$, therefore $f^{n}(z) \rightarrow x^{*}$ and $f^{n}(x) \rightarrow x^{*}$.

Of course, $x^{*}$ is the unique fixed point of $f$.

More generally, we can prove the following

Theorem 4.2. Let $X$ be a Hausdorff topological space with a partial ordering $\leq$ and let $f: X \longrightarrow X$ be an operator. Suppose that:

- One of the following conditions is satisfied:

either $f$ is orbitally continuous, or $f$ is orbitally $\leq$-continuous, and if $\left(x_{m}\right)_{m \in \mathbb{N}} \rightarrow x^{*}$ is such that $\left(x_{m}, x_{m+1}\right) \in X_{\leq}, \forall m \in \mathbb{N}$, then there exists $\left(x_{m_{k}}\right)_{k \in \mathbb{N}}$ a subsequence of $\left(x_{m}\right)_{m \in \mathbb{N}}$ such that $\left(x_{m_{k}}, x^{*}\right) \in X_{\leq}, \forall k \in \mathbb{N}$.

- $X_{\leq} \in I(f \times f)$.

- There exists $x_{0} \in X$ with $\left(x_{0}, f\left(x_{0}\right)\right) \in X_{\leq}$.

- If $\left(x_{m}\right)_{m \in \mathbb{N}}$ is a sequence in $X$ such that $\left(x_{m}, x_{m+1}\right) \in X_{\leq}, \forall m \in \mathbb{N}$, then $\left(f\left(x_{m}\right)\right)_{m \in \mathbb{N}}$ is a convergent sequence.

Then there exists at least one fixed point of $f$ in $X$. Besides, if $\mathrm{A}), \mathrm{D})$ in Theorem 3.3 hold, then $f$ is a $P O$.

Proof. Since $\left(x_{0}, f\left(x_{0}\right)\right) \in X_{\leq}$, we deduce that $\left(f^{n-1}\left(x_{0}\right)\right)_{n \in \mathbb{N}}$ is a sequence such that $\left(f^{m-1}\left(x_{0}\right), f^{m}\left(x_{0}\right)\right) \in X_{\leq}, \forall m$, then $\left(f^{n}\left(x_{0}\right)\right)_{n \in \mathbb{N}} \rightarrow x^{*}$. If $f$ is orbitally continuous, then $f\left(x^{*}\right)=x^{*}$. If not, there exists a subsequence $\left(f^{n_{k}}\left(x_{0}\right)\right)_{k \in \mathbb{N}} \rightarrow x^{*}$ such that $\left(f^{n_{k}}\left(x_{0}\right), x^{*}\right) \in X_{\leq}, \forall k$, and, by hypothesis, $\left(f^{n_{k}+1}\left(x_{0}\right)\right)_{k \in \mathbb{N}} \rightarrow f\left(x^{*}\right)$, which implies $f\left(x^{*}\right)=x^{*}$. The rest of the proof follows similarly to the proof of the previous theorem.

Theorem 4.2 is a particular case of Theorem 3.3 , and it is easy to prove that, considering the weaker condition

if $x \in X$ and $\left(f^{n}(x)\right)_{n \in \mathbb{N}}$ is a sequence such that

$$
\left(f^{n}(x), f^{n+1}(x)\right) \in X_{\leq}, \forall n, \text { then }\left(f^{n+1}(x)\right)_{n \in \mathbb{N}} \text { is convergent, }
$$

then Theorem 4.2 is an extension of Theorem 3.1

Finally, we present a result in ordered topological spaces. We recall that an ordered topological space $(X, \leq)$ is a topological space equipped with a partial ordering $\leq$ such that for each $x \in X$ the sets

$$
\{y \in X: y \leq x\} \text { and }\{y \in X: y \geq x\}
$$

are closed.

As a consequence of Proposition 1.1.3 in [7] we have that if $\left(x_{n}\right)_{n}$ is a nondecreasing convergent sequence in an ordered topological space, then

$$
\lim _{n \rightarrow \infty} x_{n}=\sup _{n \in \mathbb{N}} x_{n}
$$


and an analogous conclusion is true for nonincreasing sequences replacing sup by inf. In particular, in every ordered topological space monotone and convergent sequences have a unique limit.

The following result complements Proposition 1.2.2 in [7]:

Theorem 4.3. Let $X$ be a subset of an ordered topological space $(Y, \leq)$ and let $f: X \rightarrow X$ be an operator.

Suppose that there exists $x_{0} \in X$ such that $\left(x_{0}, f\left(x_{0}\right)\right) \in X_{\leq}$and that the following conditions hold:

- $f$ is nondecreasing on the set $C_{x_{0}}:=\left\{x \in X:\left(x, x_{0}\right) \in X_{\leq}\right\}$;

- $\left(f\left(x_{n}\right)\right)_{n}$ converges in $Y$ whenever $\left(x_{n}\right)_{n}$ is a monotone sequence in $C_{x_{0}}$;

- for all $x \in X$ the relations

$$
f^{n}(x) \rightarrow a \quad \text { and }\left(f^{n}(x)\right)_{n} \text { monotone }
$$

imply $a \in X$ and

$$
\begin{aligned}
& a \geq f(a) \quad \text { if }\left(f^{n}(x)\right)_{n} \text { is nondecreasing, } \\
& a \leq f(a) \quad \text { if }\left(f^{n}(x)\right)_{n} \text { is nonincreasing. }
\end{aligned}
$$

Then $f$ has at least one fixed point in $C_{x_{0}}$. If A), D) in Theorem 3.3 hold, then $f$ is a $P O$.

Proof. Suppose that $x_{0} \leq f\left(x_{0}\right)$; then $f\left(x_{0}\right) \in C_{x_{0}}$ and hence $f\left(x_{0}\right) \leq f^{2}\left(x_{0}\right)$ because $f$ is nondecreasing on $C_{x_{0}}$. Inductively one can show that $f^{n}\left(x_{0}\right) \in C_{x_{0}}$ for all $n \in \mathbb{N}$ and, moreover, that

$$
x_{0} \leq f^{n}\left(x_{0}\right) \leq f^{n+1}\left(x_{0}\right) \text { for all } n \in \mathbb{N} .
$$

Therefore $\left(f^{n-1}\left(x_{0}\right)\right)_{n}$ is nondecreasing and then $\left(f^{n}\left(x_{0}\right)\right)_{n}$ is convergent to some $x^{*} \in Y$ that satisfies

$$
f^{n}\left(x_{0}\right) \leq x^{*} \quad \text { for all } n \in \mathbb{N} \text {. }
$$

Moreover $\left(f^{n}\left(x_{0}\right)\right)_{n}$ is nondecreasing and converges to $x^{*}$, which implies that $x^{*} \in X$ and

$$
f\left(x^{*}\right) \leq x^{*} .
$$

Now from (4.1) and (4.2) we deduce that $x_{0} \leq x^{*}$ (in particular, $x^{*} \in C_{x_{0}}$ ), thus

$$
f^{n}\left(x_{0}\right) \leq f\left(x^{*}\right) \leq x^{*} \text { for all } n \in \mathbb{N}
$$

hence $x^{*} \leq f\left(x^{*}\right) \leq x^{*}$, i.e., $x^{*}$ is a fixed point of $f$.

Remark 4.4. Theorem 4.3 is also valid for ordered $L$-spaces satisfying (1.1) and (1.2).

\section{Applications}

Consider the set $X$ given by

$$
\begin{aligned}
X=P C(J):=\{x: J \longrightarrow & \mathbb{R}: x \text { is continuous in }[0, T) \backslash\left\{t_{k}: k \in \mathbb{N}\right\}, \text { and } \\
& \text { there exist } \left.x\left(t_{k}^{-}\right), k \in \mathbb{N}, x\left(t_{k}^{+}\right)=x\left(t_{k}\right), k \in \mathbb{N} \cup\{0\}\right\},
\end{aligned}
$$

where $T>0, J=[0, T)$, and $0=t_{0}<t_{1}<t_{2}<\cdots<t_{k}<t_{k+1}<\cdots<T$ are such that $\lim _{k \rightarrow+\infty} t_{k}=T$. Denote by $J_{k}=\left[t_{k}, t_{k+1}\right]$, for $k \in \mathbb{N} \cup\{0\}$.

Given $x \in P C(J)$, we define $x_{k} \in C\left(J_{k}\right), k=0,1,2, \ldots$, by

$$
x_{k}(t)=x(t), t \in\left[t_{k}, t_{k+1}\right), x_{k}\left(t_{k+1}\right)=x\left(t_{k+1}^{-}\right) .
$$


Reciprocally, for a family of functions $\left\{x_{k}: x_{k} \in C\left(J_{k}\right), k \in \mathbb{N} \cup\{0\}\right\}$, we define $x \in P C(J)$ as $x(t)=x_{k}(t)$, for $t \in\left[t_{k}, t_{k+1}\right), k=0,1,2, \ldots$ Hence the space $P C(J)$ can be interpreted as the infinite product $\prod_{k \in \mathbb{N} \cup\{0\}} C\left(J_{k}\right)$.

$P C(J)$ has structure of $L$-space, if we consider $c(X)$ the set of sequences $\left(x_{n}\right)_{n \in \mathbb{N}}$ in $X=P C(J)$ such that the sequence $\left(\left(x_{n}\right)_{k}\right)_{n \in \mathbb{N}}$ is convergent in $C\left(J_{k}\right)$, for every $k=0,1,2, \ldots$, and $\operatorname{Lim}: c(X) \longrightarrow X$ is the mapping given by $\left(\operatorname{Lim}\left(x_{n}\right)\right)_{k}=$ $\lim _{n \rightarrow+\infty}\left(x_{n}\right)_{k} \in C\left(J_{k}\right)$, for $\left(x_{n}\right)_{n \in \mathbb{N}} \in c(X)$ and $k=0,1,2, \ldots$ Indeed, we check properties i), ii) in Definition 2.2.

- If $x_{n}=x$, for all $n \in \mathbb{N}$, then $\left(x_{n}\right)_{k}=x_{k} \in C\left(J_{k}\right)$, for all $n \in \mathbb{N}$ and $k \in \mathbb{N} \cup\{0\}$, then $\left(\left(x_{n}\right)_{k}\right)_{n \in \mathbb{N}} \rightarrow x_{k} \in C\left(J_{k}\right)$, for every $k=0,1,2, \ldots$, and $\left(x_{n}\right)_{n \in \mathbb{N}} \in c(X)$ with $\operatorname{Lim}\left(x_{n}\right)_{n \in \mathbb{N}}=x$.

- If $\left(x_{n}\right)_{n \in \mathbb{N}} \in c(X)$ and $\operatorname{Lim}\left(x_{n}\right)_{n \in \mathbb{N}}=x$, then $\left(\left(x_{n}\right)_{k}\right)_{n \in \mathbb{N}} \in c\left(C\left(J_{k}\right)\right)$, satisfying $\left(\left(x_{n}\right)_{k}\right)_{n \in \mathbb{N}} \rightarrow x_{k}$, for every $k=0,1, \ldots$ This implies that, for every subsequence $\left(x_{n_{i}}\right)_{i \in \mathbb{N}}$ of $\left(x_{n}\right)_{n \in \mathbb{N}}$ we have that $\left(\left(x_{n_{i}}\right)_{k}\right)_{i \in \mathbb{N}} \rightarrow x_{k}$, for $k=0,1, \ldots$, obtaining $\left(x_{n_{i}}\right)_{i \in \mathbb{N}} \in c(X)$ and $\operatorname{Lim}\left(x_{n_{i}}\right)_{i \in \mathbb{N}}=x$.

Moreover, $(P C(J), \rightarrow, \leq)$ is an ordered $L$-space. Indeed, $P C(J)$ is a partially ordered set considering the partial ordering $\leq$ given by

$$
x \leq y \Longleftrightarrow x(t) \leq y(t), \text { for every } t \in J,
$$

which is equivalent to $x_{k} \leq y_{k}$ in $J_{k}$, for every $k=0,1,2, \ldots$, and, besides, for $\left(x_{n}\right)_{n \in \mathbb{N}} \rightarrow x,\left(y_{n}\right)_{n \in \mathbb{N}} \rightarrow y$ such that $x_{n} \leq y_{n}$, for each $n \in \mathbb{N}$, we get $\left(x_{n}\right)_{k} \leq\left(y_{n}\right)_{k}$ in $C\left(J_{k}\right)$, for each $n \in \mathbb{N}, k \in \mathbb{N} \cup\{0\}$, and $\left(\left(x_{n}\right)_{k}\right)_{n \in \mathbb{N}} \rightarrow x_{k},\left(\left(y_{n}\right)_{k}\right)_{n \in \mathbb{N}} \rightarrow y_{k}, \forall k=$ $0,1,2, \ldots$. This implies that $x_{k} \leq y_{k}$, for every $k$ and, therefore, $x \leq y$.

Note that $d(x, y)=\sup _{t \in J} d(x(t), y(t))=\sup _{k \in \mathbb{N} \cup\{0\}} \sup _{t \in J_{k}}\left|x_{k}(t)-y_{k}(t)\right|$, for $x, y \in$ $X$, is not necessarily a finite number. However, $d$ defines a metric in the space $P C_{M}(J):=\{x \in P C(J):-M \leq x(t) \leq M, \forall t \in J\}$, where $M>0$, since, for $x, y \in P C_{M}(J),\left|x_{k}(t)-y_{k}(t)\right| \leq 2 M$, for $t \in J_{k}$, and $k=0,1, \ldots$ The space $P C_{M}(J)$ is an ordered $L$-space as well.

For the ordered $L$-space $(P C(J), \rightarrow, \leq)$, condition A) in Theorem 3.3 holds. Indeed, for every $x, y \in P C(J)$, it is possible to define $\max \{x, y\}, \min \{x, y\} \in$ $P C(J)$, respectively, by $(\max \{x, y\})_{k}=\max \left\{x_{k}, y_{k}\right\} \in C\left(J_{k}\right)$ and $(\min \{x, y\})_{k}=$ $\min \left\{x_{k}, y_{k}\right\} \in C\left(J_{k}\right)$, for $k=0,1,2, \ldots$ Obviously, $\min \{x, y\} \leq x, y \leq \max \{x, y\}$, since it is satisfied on each $J_{k}$.

Following the proof of Theorem 3.3. we deduce the existence of fixed points for orbitally-continuous mappings $F: P C(J) \rightarrow P C(J)$ under the hypothesis of existence of $x_{0} \in P C(J)$ with $\left(F^{n}\left(x_{0}\right)\right)_{n}$ convergent. This kind of fixed point result is useful to obtain the existence of solution for equations defined in the space $P C(J)$, which allows the impulsive behavior of the solution at an infinite number of points in the interval $J$, that is, the solution could be a function with an infinite number of discontinuities $t_{k}$ accumulating at $t=T$, and, therefore, $\inf \left\{t_{k}-t_{k-1}\right.$ : $k \in \mathbb{N}\}=0$. This case has not been discussed in the literature as much as the case with a finite number of discontinuities $t_{k}$, due to the particular behavior of the elements in $P C(J)$. Thus, the development of new fixed point theorems valid in more general spaces arises as an interesting point. For instance, $L$-spaces and fixed point theorems in $L$-spaces provide suitable tools for the analysis of existence and uniqueness of solutions for differential and integro-differential equations with underlying space $P C(J)$. Besides, some results in [1,4] concerning matrix equations 
can be extended by taking into account that our results consider some weaker hypotheses with respect to the fixed point results in those references.

We present an example in the context of integral equations, where solutions have an infinite number of jumps in the bounded interval $J$. Consider the integral equation

$$
x(t)=\max \left\{-M, \min \left\{h(t)+\int_{t_{k}}^{t} G(t, s) f(s, x(s)) d s, M\right\}\right\}, t \in\left[t_{k}, t_{k+1}\right),
$$

where $k=0,1,2, \ldots, M>0, h \in P C(J), G \in P C\left(\Delta, \mathbb{R}^{+}\right), G$ bounded, where $\Delta:=\bigcup_{k=0}^{+\infty}\left\{(t, s) \in \mathbb{R}^{2}: t_{k} \leq s \leq t \leq t_{k+1}\right\}$, and $f: J \times \mathbb{R} \longrightarrow \mathbb{R}$ is continuous in $\left(t_{k}, t_{k+1}\right) \times \mathbb{R}, k=0,1,2, \ldots$, and such that there exist the $\operatorname{limits}_{\lim } \operatorname{lit}_{k}^{+} f(t, x)=$ $f\left(t_{k}, x\right)$, for every $x \in \mathbb{R}, k=0,1,2, \ldots$, and $\lim _{t \rightarrow t_{k}^{-}} f(t, x)$, for $x \in \mathbb{R}, k=1,2, \ldots$

Theorem 5.1. Suppose that the following conditions hold:

(H1): $f$ is nondecreasing in the second variable: $f(t, x) \leq f(t, y), \forall t \in J, x \leq$ $y \in \mathbb{R}$.

(H2): If $\left(y_{n}\right) \subseteq P C_{M}(J)$ is monotone and $\left(y_{n}\right) \rightarrow a$ in $P C_{M}(J)$, then the sequence $\left(f\left(\cdot, y_{n}(\cdot)\right)\right) \subseteq P C(J)$ is such that $\sup _{t \in J}\left|f\left(t, y_{n}(t)\right)-f(t, a(t))\right| \rightarrow 0$, as $n \rightarrow+\infty$.

(H3): There exists $x_{0} \in P C_{M}(J)$ with $x_{0}(t) \leq h(t)+\int_{t_{k}}^{t} G(t, s) f\left(s, x_{0}(s)\right) d s$, $\forall t \in J\left(\right.$ or $\left.x_{0}(t) \geq h(t)+\int_{t_{k}}^{t} G(t, s) f\left(s, x_{0}(s)\right) d s, \forall t \in J\right)$.

(H4): If $\left(y_{n}\right) \subseteq P C_{M}(J)$ is monotone, then

$$
\left(\max \left\{-M, \min \left\{h(t)+\int_{t_{k}}^{t} G(t, s) f\left(s,\left(y_{n}\right)_{k}(s)\right) d s, M\right\}\right\}\right)
$$

is convergent in $C\left(J_{k}\right)$, uniformly in $k=0,1,2, \ldots$

Then there exists at least one solution of equation (5.1).

Proof. Define the operator $F: P C_{M}(J) \longrightarrow P C_{M}(J)$, where $(F x)(t)$, for $x \in$ $P C_{M}(J)$ and $t \in J$, is given by the right-hand side in (5.1). It is easy to check that hypotheses in Theorem 4.1 are valid and, thus, there exists at least one fixed point of $F$ in $P C_{M}(J)$, that is, a solution of equation (5.1) which lies in the functional interval $[-M, M]$.

This result can be extended and the existence of solution to (5.1) can be derived from Theorem 4.3 , since $P C_{M}(J)$ is an ordered topological space. One of the improvements is that hypotheses (H1) and (H4) can be weakened to its validity in the set $C_{x_{0}}:=\left\{x \in P C_{M}(J): x \leq x_{0}\right.$ or $\left.x \geq x_{0}\right\}$, also obtaining a solution in the set $C_{x_{0}}$.

\section{ACKNOWLEDGMENTS}

The authors thank the editor and the referee for their useful remarks and interesting comments.

\section{REFERENCES}

1. A.C.M. Ran, M.C.B. Reurings, A fixed point theorem in partially ordered sets and some applications to matrix equations, Proc. Amer. Math. Soc. 132 (2004), 1435-1443. MR2053350 (2005a:47112) 
2. J.J. Nieto, R. Rodríguez-López, Contractive Mapping Theorems in Partially Ordered Sets and Applications to Ordinary Differential Equations, Order, 22 (2005), 223-239. MR.2212687

3. J.J. Nieto, R. Rodríguez-López, Existence and Uniqueness of Fixed Point in Partially Ordered Sets and Applications to Ordinary Differential Equations, Acta Math. Sinica (English Series), to appear.

4. A. Petrussel, I.A. Rus, Fixed point theorems in ordered L-spaces, Proc. Amer. Math. Soc. 134 (2006), 411-418. MR2176009 (2006g:47097)

5. M. Fréchet, Les espaces abstraits, Gauthiers-Villars, Paris, 1928.

6. P. Diamond, P.E. Kloeden, Metric spaces of fuzzy sets: theory and applications, World Scientific, Singapore, 1994. MR1337027(96e:54003)

7. S. Heikkilä, V. Lakshmikantham, Monotone iterative techniques for discontinuous nonlinear differential equations, Marcel Dekker, New York, 1994. MR1280028 (95d:34002)

Departamento de Análisis Matemático, Facultad de Matemáticas, Universidad de Santiago de Compostela, 15782, Spain

E-mail address: amnieto@usc.es

Departamento de Análisis Matemático, Facultad de Matemáticas, Universidad de Santiago de Compostela, 15782, Spain

E-mail address: rodrigolp@usc.es

Departamento de Análisis Matemático, Facultad de Matemáticas, Universidad de Santiago de Compostela, 15782, Spain

E-mail address: amrosana@usc.es 